\begin{tabular}{|c|l|}
\hline Title & Double Commuting Compressed Shifts and Generalized Interpolation in the Hardy Space over the Bidisk \\
\hline Author(s) & Nakazi, Takahiko; Seto, Michio \\
\hline Citation & $\begin{array}{l}\text { Integral Equations and Operator Theory, 56(4), 543-558 } \\
\text { https://doi.org/40.1007/300020-006-1432-6 }\end{array}$ \\
\hline Issue Date & $2006-12$ \\
\hline Doc URL & http://hdl.handle.net/2115/18847 \\
\hline Rights & The original publication is available at www.springerlink.com \\
\hline Type & article (author version) \\
\hline File Information & IEOT56-4.pdf \\
\hline
\end{tabular}

Instructions for use 


\title{
Double commuting compressed shifts and gen- eralized interpolation in the Hardy space over the bidisk
}

\author{
Takahiko Nakazi and Michio Seto
}

\begin{abstract}
This paper deals with an operator theory of compressed shifts on the Hardy space over the bidisk. We give commutant lifting type theorems and some interpolation theorems in two variables.
\end{abstract}

Mathematics Subject Classification (2000). Primary 47B38; Secondary 47B35.

Keywords. Hardy spaces, compressed shifts, commutant lifting theorem and interpolation problems.

\section{Introduction}

In [8], D. Sarason gave an operator theory approach to classical interpolation problems of complex function theory, Carathéodory interpolation problem and Nevanlinna-Pick interpolation problem, in the Hardy space over the unit disk. It shows a deep connection between operator theory and complex function theory. Sz.-Nagy and Foiaş generalized Sarason's technique as their commutant lifting theorem for a contraction on an abstract Hilbert space in [9] (cf. [1] and [7]). It is one of the most useful tools in operator theory, so that one would attempt to find a commutant lifting theorem for tuples of contractions. However, for tuples of contractions, there are examples in which the commutant lifting theorem fails.

Clark [4] and Amar-Menini [2] gave interesting counter-examples of the commutant lifting theorem in the Hardy space over the polydisk. Their examples are tuples of compressed shifts of coordinate functions. In particular, Amar-Menini dealt with the bidisk. The authors have been motivated to study commutant lifting type theorems in the Hardy space over the bidisk by their example. Our interest is the following question:

This research was supported by 21st Century COE Program, "Mathematics of Nonlinear Structures via Singularities", Department of Mathematics, Hokkaido University. 
- Why does the commutant lifting theorem fail for compressed shifts in the Hardy space over the bidisk?

It seems to be one of the first steps toward answering the above question that we study non-trivial cases where commutant lifting type theorems hold for compressed shifts.

In this paper, we attempt to find non-trivial commutant lifting type theorems for compressed shifts as a sequel to [5]. Section 2 introduces notions used in this paper. In Section 3, we shall prove commutant lifting type theorems in two variables. In Section 4, we deal with norm estimates of compressed operators. In Section 5, a question related to commutant lifting is posed. In Sections 6 and 7, we deal with Carathéodory type interpolation and Nevanlinna-Pick type interpolation problems in two variables, respectively.

\section{Preliminaries}

Let $\mathbb{D}=\{\lambda \in \mathbb{C}:|\lambda|<1\}$, the unit disk in the complex plane $\mathbb{C}$ and let $\mathbb{T}=\left\{e^{i \theta} \in\right.$ $\mathbb{C}: 0 \leq \theta<2 \pi\}$, the unit circle in $\mathbb{C} . L^{2}=L^{2}\left(\mathbb{T}^{2}\right)$ denotes the Hilbert space of all square integrable functions with respect to the normalized Lebesgue measure $d \sigma$ on $\mathbb{T}^{2}$. For a closed subspace $\mathcal{M}$ of $L^{2}, P_{\mathcal{M}}$ denotes the orthogonal projection from $L^{2}$ onto $\mathcal{M}$. $L^{\infty}=L^{\infty}\left(\mathbb{T}^{2}\right)$ denotes the commutative Banach algebra of all essentially bounded measurable functions with respect to the measure $d \sigma$ on $\mathbb{T}^{2}$. $H^{2}=H^{2}\left(\mathbb{D}^{2}\right)$ will denote the Hardy space over the bidisk $\mathbb{D}^{2}$ in $\mathbb{C}^{2}, H^{2}$ consists of all analytic functions on $\mathbb{D}^{2}$ satisfying the following condition:

$$
\sup _{0 \leq r<1} \int_{\mathbb{T}} \int_{\mathbb{T}}|f(r z, r w)|^{2} d \sigma<+\infty,
$$

where $z$ and $w$ are the usual coordinate functions on $\mathbb{C}^{2}$. Let $H^{2}(z)$ (resp. $H^{2}(w)$ ) denote the usual one-variable Hardy space over $\mathbb{D}$ of the variable $z$ (resp. $w$ ) with respect to the normalized Lebesgue measure $d \sigma_{1}$ (resp. $\left.d \sigma_{2}\right)$ on $\mathbb{T}$. Then it is well known that $H^{2}=H^{2}(z) \otimes H^{2}(w)$, the Hilbert space tensor product of $H^{2}(z)$ and $H^{2}(w) . H^{\infty}=H^{\infty}\left(\mathbb{D}^{2}\right)$ will denote the commutative Banach algebra consisting of all bounded analytic functions on $\mathbb{D}^{2}$ with the norm $\|f\|_{\infty}=\sup _{\lambda, \mu \in \mathbb{D}}|f(\lambda, \mu)|$. The Toeplitz operator $T_{\varphi}$ on $H^{2}$ of a bounded analytic function $\varphi$ is the restriction of the multiplication operator of $\varphi$ on $H^{2}$.

Definition 2.1. A closed subspace $\mathcal{N}$ of $H^{2}$ is called a backward shift invariant subspace if $\mathcal{N}$ is invariant under $T_{z}^{*}$ and $T_{w}^{*}$. For a bounded analytic function $\varphi$, $S_{\varphi}$ denotes the compression of $T_{\varphi}$ in a backward shift invariant subspace $\mathcal{N}$, that is, $S_{\varphi} f=P_{\mathcal{N}} \varphi f$ for any $f$ in $\mathcal{N}$. In particular, $S_{z}$ and $S_{w}$ are called compressed shifts.

$\mathbb{C}[z, w]$ will denote the polynomial ring over $\mathbb{C}$ with variables $z$ and $w$. Every backward shift invariant subspace $\mathcal{N}$ has a module structure over $\mathbb{C}[z, w]$ by the action defined as follows: $f \cdot h=S_{f} h=P_{\mathcal{N}} f h$ for any $f$ in $\mathbb{C}[z, w]$ and $h$ in $\mathcal{N}$.

In [5], Izuchi and the authors showed the following theorem: 
Theorem 2.1 ([5]). If a backward shift invariant subspace $\mathcal{N}$ satisfies the condition $\left[S_{z}^{*}, S_{w}\right]=S_{z}^{*} S_{w}-S_{w} S_{z}^{*}=0$, then one and only one of the following occurs:

(i) $\mathcal{N}=H^{2} \ominus q_{1}(z) H^{2}=\left(H^{2}(z) \ominus q_{1}(z) H^{2}(z)\right) \otimes H^{2}(w)$,

(ii) $\mathcal{N}=H^{2} \ominus q_{2}(w) H^{2}=H^{2}(z) \otimes\left(H^{2}(w) \ominus q_{2}(w) H^{2}(w)\right)$,

(iii) $\mathcal{N}=\left(H^{2}(z) \ominus q_{1}(z) H^{2}(z)\right) \otimes\left(H^{2}(w) \ominus q_{2}(w) H^{2}(w)\right)$.

where $q_{1}(z)$ and $q_{2}(w)$ are one-variable inner functions.

In this paper, we deal mainly with operators on the tensor product Hilbert space $\mathcal{N}$ obtained in Theorem 2.1, and use the notation $\mathcal{N}=\mathcal{N}_{z} \otimes \mathcal{N}_{w}$, for short.

\section{Commutant lifting type theorems for $S_{z}$ and $S_{w}$}

Let $\mathcal{A}$ be the weak closed commutative Banach algebra generated by $S_{z}, S_{w}$ and the identity operator $I_{\mathcal{N}}$ on a backward shift invariant subspace $\mathcal{N}$, and let $\mathcal{A}^{\prime}$ denote the commutant of $\mathcal{A}$ in the Banach algebra of all bounded linear operators on $\mathcal{N}$.

Regarding the algebra $\mathcal{A}$ on a backward shift invariant subspace $\mathcal{N}$, we observe the following statements:

(A) $\mathcal{A}^{\prime}=\left\{S_{\varphi}: \varphi \in H^{\infty}\right\}$,

(B) $\mathcal{A}=\mathcal{A}^{\prime}$

(C) $\mathcal{A}^{\prime}=\mathcal{A}^{\prime \prime}$, where $\mathcal{A}^{\prime \prime}$ is the double commutant of $\mathcal{A}$.

First, we note that $\mathcal{A}$ is equal to the weak closure of the set $\left\{S_{\varphi}: \varphi \in H^{\infty}\right\}$ and the statement $(\mathrm{B})$ is equivalent to the following $\left(\mathrm{B}^{\prime}\right)$ :

$\left(\mathrm{B}^{\prime}\right)$ for any operator $A$ in $\mathcal{A}^{\prime}$, there exists a sequence $\left\{\varphi_{n}\right\}$ in $H^{\infty}$ such that $S_{\varphi_{n}}$ converges to $A$ in the weak operator topology.

Indeed, for any function $\varphi$ in $H^{\infty}$, there exists a sequence $\left\{p_{n}\right\}$ in $\mathbb{C}[z, w]$ such that $p_{n}$ converges to $\varphi$ in the $\mathrm{w}^{*}$-topology. Then $\left\langle S_{p_{n}} f, g\right\rangle$ converges to $\left\langle S_{\varphi} f, g\right\rangle$ for any functions $f$ and $g$ in $\mathcal{N}$. This implies that $\mathcal{A}$ is equal to the weak closure of the set $\left\{S_{\varphi}: \varphi \in H^{\infty}\right\}$. Hence (B) is equivalent to $\left(\mathrm{B}^{\prime}\right)$. Further, it is easy to see that $(\mathrm{A}) \Rightarrow(\mathrm{B}) \Rightarrow(\mathrm{C})$ and $(\mathrm{A})$ is the straightforward version of the Sarason theorem in the bidisk. However, Amar and Menini gave a counter-example of $(\mathrm{A})$ in $[2]$.

In this section, first, we prove lemmas needed later. Next, we will prove (C) on $\mathcal{N}=\mathcal{N}_{z} \otimes \mathcal{N}_{w}$ in Theorem 3.1, and we will give examples where (B) and (A) hold in Theorems 3.2 and 3.3, respectively.

The following Lemma 3.1 is known in [3]. For the sake of reader's convenience, we prove this in our setting.

Lemma 3.1. If $A$ is in $\mathcal{A}^{\prime}$ on $\mathcal{N}=\mathcal{N}_{z} \otimes \mathcal{N}_{w}$, then there exists a function $\Phi$ in $\mathcal{N}$ such that $A=S_{\Phi}$, where $S_{\Phi}$ is the bounded operator on $\mathcal{N}$ defined by the following identity: $S_{\Phi} f=P_{\mathcal{N}} \Phi f$ for any $f$ in $\mathcal{N} \cap H^{\infty}$. 
Proof . Let $A P_{\mathcal{N}} 1=\Phi$, and let $f(z)$ and $g(w)$ be bounded analytic functions in $\mathcal{N}_{z}$ and $\mathcal{N}_{w}$, respectively. Then we have

$$
\begin{aligned}
A f(z) g(w) & =A S_{f(z)} S_{g(w)} P_{\mathcal{N} 1} \\
& =S_{f(z)} S_{g(w)} A P_{\mathcal{N} 1} \\
& =S_{f(z)} S_{g(w)} \Phi \\
& =P_{\mathcal{N}} f(z) g(w) \Phi \\
& =S_{\Phi} f(z) g(w) .
\end{aligned}
$$

Since $\mathcal{N} \cap H^{\infty}$ is dense in $\mathcal{N}$, we have $A=S_{\Phi}$.

Note that $\Phi$ may be an unbounded function in Lemma 3.1. However $S_{\Phi}$ is a bounded operator on $\mathcal{N}_{z} \otimes \mathcal{N}_{w}$. We set $\Phi_{r}(z, w)=\Phi(r z, r w)$ for $0 \leq r<1$. Then it is known that $\Phi_{r}$ is a bounded analytic function converging to $\Phi$ as $r$ tends to 1 in the $L^{2}$-norm topology. Setting

$$
\mathcal{D}=\mathcal{D}(T)=\left\{f \in \mathcal{N}: \sup _{0 \leq r<1}\left\|S_{\Phi_{r}} f\right\|<\infty\right\},
$$

a densely defined linear operator $T$ can be defined as follows:

$$
T h=\lim _{r \rightarrow 1-0} S_{\Phi_{r}} h
$$

in the weak topology, where $h$ is any function in $\mathcal{D}$. Trivially, $S_{\Phi}$ is the closed extension of $T$.

Lemma 3.2. We set $\xi=P_{\mathcal{N}} 1$. Then $\xi$ is a cyclic separating vector for $\mathcal{A}^{\prime}$ on $\mathcal{N}=\mathcal{N}_{z} \otimes \mathcal{N}_{w}$. Moreover $\xi$ is a bounded analytic function.

Proof . It is easy to show that $\xi=P_{\mathcal{N}} 1=\left(1-\overline{q_{1}(0)} q_{1}(z)\right)\left(1-\overline{q_{2}(0)} q_{2}(w)\right)$, which is a bounded analytic function. First, we show $\xi$ is a cyclic vector for $\mathcal{A}^{\prime}$. If there exists a function $f$ in $\mathcal{N}$ such that $\left\langle S_{z}^{k} S_{w}^{l} \xi, f\right\rangle=0$ for any non-negative integers $k$ and $l$, then we have $f=0$. Indeed, we have

$$
\left\langle z^{k} w^{l}, f\right\rangle=\left\langle S_{z}^{k} S_{w}^{l} \xi, f\right\rangle=0,
$$

which implies that $f=0$. Hence $\xi$ is a cyclic vector for $\mathcal{A}$. Since $\mathcal{A}$ is contained in $\mathcal{A}^{\prime}, \xi$ is cyclic for $\mathcal{A}^{\prime}$. Further, if $A \xi=0$ for an operator $A$ in $\mathcal{A}^{\prime}$ then $A S_{z}^{k} S_{w}^{l} \xi=$ $S_{z}^{k} S_{w}^{l} A \xi=0$. Since $\xi$ is cyclic for $\mathcal{A}^{\prime}$, we have $A=0$. This completes the proof.

Let $K_{\lambda, \mu}$ be the Cauchy kernel of $H^{2}$ at $(\lambda, \mu)$ in $\mathbb{D}^{2}$. We set

$$
\xi_{\lambda, \mu}=P_{\mathcal{N}} K_{\lambda, \mu}=\frac{\left(1-\overline{q_{1}(\lambda)} q_{1}(z)\right)\left(1-\overline{q_{2}(\mu)} q_{2}(w)\right)}{(1-\bar{\lambda} z)(1-\bar{\mu} w)},
$$

where $\mathcal{N}=\mathcal{N}_{z} \otimes \mathcal{N}_{w}$. Trivially, every $\xi_{\lambda, \mu}$ is a bounded analytic function. Further, it is easy to check that $\xi_{0,0}=\xi$ and $\xi_{\lambda, \mu}=S_{K_{\lambda, \mu}} \xi$. It follows that every $\xi_{\lambda, \mu}$ is a cyclic separating vector for $\mathcal{A}^{\prime}$ by Lemma 3.2 . 
Lemma 3.3. Let $\mathcal{N}=\mathcal{N}_{z} \otimes \mathcal{N}_{w}$. If $A$ is in $\mathcal{A}^{\prime}$ such that $A=S_{\Phi}$ for some function $\Phi$ in $\mathcal{N}$, then $S_{\Phi_{r}}^{*} \xi_{\lambda, \mu}$ converges to $A^{*} \xi_{\lambda, \mu}$ as $r$ tends to 1 in the $L^{2}$-norm topology for any $(\lambda, \mu)$ in $\mathbb{D}^{2}$.

Proof . First we show that $A^{*} \xi_{\lambda, \mu}=P_{\mathcal{N}} \bar{\Phi} \xi_{\lambda, \mu}$. For any bounded function $f$ in $\mathcal{N}$, we have

$$
\left\langle A^{*} \xi_{\lambda, \mu}, f\right\rangle=\left\langle\xi_{\lambda, \mu}, A f\right\rangle=\left\langle\xi_{\lambda, \mu}, P_{\mathcal{N}} \Phi f\right\rangle=\int \xi_{\lambda, \mu} \overline{\Phi f} d \sigma=\left\langle P_{\mathcal{N}} \bar{\Phi} \xi_{\lambda, \mu}, f\right\rangle .
$$

Since $\mathcal{N} \cap H^{\infty}$ is dense in $\mathcal{N}$, we have that $A^{*} \xi_{\lambda, \mu}=P_{\mathcal{N}} \bar{\Phi} \xi_{\lambda, \mu}$. Using this, we obtain that

$$
\begin{aligned}
\left\|S_{\Phi_{r}}^{*} \xi_{\lambda, \mu}-A^{*} \xi_{\lambda, \mu}\right\| & =\left\|P_{\mathcal{N}} \overline{\Phi_{r}} \xi_{\lambda, \mu}-P_{\mathcal{N}} \bar{\Phi} \xi_{\lambda, \mu}\right\| \\
& \leq\left\|\bar{\Phi}_{r} \xi_{\lambda, \mu}-\bar{\Phi} \xi_{\lambda, \mu}\right\| \\
& \leq\left\|\bar{\Phi}_{r}-\bar{\Phi}\right\|\left\|\xi_{\lambda, \mu}\right\|_{\infty} \rightarrow 0
\end{aligned}
$$

as $r$ tends to 1 . This concludes the proof.

Theorem 3.1. If $\mathcal{N}=\mathcal{N}_{z} \otimes \mathcal{N}_{w}$, then $\mathcal{A}^{\prime}$ is commutative, that is, $\mathcal{A}^{\prime}=\mathcal{A}^{\prime \prime}$.

Proof . If $A$ and $B$ are any elements in $\mathcal{A}^{\prime}$, then it suffices to show $A B \xi=B A \xi$ by Lemma 3.2. Then there exists a function $\Phi$ in $\mathcal{N}$ such that $A=S_{\Phi}$ by Lemma 3.1. Further, it is easy to check that

$$
B A \xi=\lim _{r \rightarrow 1-0} B S_{\Phi_{r}} \xi=\lim _{r \rightarrow 1-0} S_{\Phi_{r}} B \xi .
$$

Hence we have

$$
\begin{aligned}
\left\langle B A \xi, \xi_{\lambda, \mu}\right\rangle & =\lim _{r \rightarrow 1-0}\left\langle S_{\Phi_{r}} B \xi, \xi_{\lambda, \mu}\right\rangle \\
& =\lim _{r \rightarrow 1-0}\left\langle B \xi, S_{\Phi_{r}}^{*} \xi_{\lambda, \mu}\right\rangle \\
& =\left\langle B \xi, A^{*} \xi_{\lambda, \mu}\right\rangle \\
& =\left\langle A B \xi, \xi_{\lambda, \mu}\right\rangle,
\end{aligned}
$$

by Lemma 3.3. Since every $\xi_{\lambda, \mu}$ is the reproducing kernel of $\mathcal{N}$ at $(\lambda, \mu)$, we have that $A B \xi=B A \xi$. This concludes the proof.

Theorem 3.2. If $A$ is in $\mathcal{A}^{\prime}$ on $\mathcal{N}=H^{2} \ominus q(z) H^{2}$, then there exists a family of bounded analytic functions $\left\{\varphi_{j}(z)\right\}$ depending only on the variable $z$ such that

$$
A=\sum_{j \geq 0} S_{\varphi_{j}(z)} T_{w}^{j}
$$

in the weak operator topology.

Proof . Since $A$ commutes with $T_{w}, A$ is an analytic function which takes values in operators on $\mathcal{N}_{z}$. Therefore, we have the following expansion of $A$ :

$$
A=\sum_{j=0}^{\infty} A_{j} \otimes T_{w}^{j} .
$$


Let $Q_{k}$ denote the orthogonal projection from $\mathcal{N}=\mathcal{N}_{z} \otimes H^{2}(w)$ onto $\mathcal{N}_{z} \otimes \mathbb{C} w^{k}$. Then $A=\sum_{k, l>0} Q_{k} A Q_{l}$ in the weak operator topology. Further, we have $Q_{k} A Q_{l}=Q_{k}\left(A_{k-l} \otimes T_{w}^{k-l}\right) Q_{l}$ for $k \geq l$. Indeed, it is easy to check the following:

$$
Q_{k} A Q_{l}=\sum Q_{k}\left(A_{j} \otimes T_{w}^{j}\right) Q_{l}=Q_{k}\left(A_{k-l} \otimes T_{w}^{k-l}\right) Q_{l} .
$$

Since $S_{z}$ commutes with every $Q_{k}$, for any function $f(z)$ in $\mathcal{N}_{z}$, we have the following:

$$
\begin{aligned}
S_{z} A_{k-l} f(z) w^{k} & =S_{z} Q_{k}\left(A_{k-l} \otimes T_{w}^{k-l}\right) Q_{l} f(z) w^{l} \\
& =Q_{k}\left(A_{k-l} \otimes T_{w}^{k-l}\right) Q_{l} S_{z} f(z) w^{l}=A_{k-l} S_{z} f(z) w^{k} .
\end{aligned}
$$

It follows that $S_{z}$ commutes with every $A_{j}$ on $\mathcal{N}_{z}$. Hence there exists a bounded analytic function $\varphi_{j}(z)$ such that $A_{j}=S_{\varphi_{j}(z)}$ by Thoerem 1 in [8]. This concludes the proof.

Corollary 3.1. $\mathcal{A}=\mathcal{A}^{\prime}$ on $\mathcal{N}=H^{2} \ominus q(z) H^{2}$.

If we assume that the dimension of $\mathcal{N}$ is finite in Lemma 3.1, then every element in $\mathcal{N}$ is a rational function. Hence it is an immediate consequence that there exists a bounded analytic function $\varphi$ such that $A=S_{\varphi}$ when $\mathcal{N}$ is of finite dimension. Then, trivially $\mathcal{A}=\mathcal{A}^{\prime}$. We show further details of this fact in the next theorem.

For $\lambda$ in $\mathbb{D}$, we define two functions as follows:

$$
b_{\lambda}(z)=(z-\lambda) /(1-\bar{\lambda} z), \quad k_{\lambda}(z)=(1-\bar{\lambda} z)^{-1} .
$$

Lemma 3.4. Let $q_{1}(z)$ be a finite Blaschke product, each zero $\lambda_{i}$ having multiplicity $k_{i}$, that $i s$,

$$
q_{1}(z)=\prod_{i=0}^{l}\left(\frac{z-\lambda_{i}}{1-\overline{\lambda_{i}} z}\right)^{k_{i}}=\prod_{i=0}^{l} b_{\lambda_{i}}^{k_{i}}(z) .
$$

Then

$$
\left\{e_{i}^{(j)}(z)=k_{\lambda_{i}}(z) b_{\lambda_{0}}^{k_{0}}(z) \cdots b_{\lambda_{i-1}}^{k_{i-1}}(z) b_{\lambda_{i}}^{j}(z): 0 \leq i \leq l, 0 \leq j \leq k_{i}-1\right\}
$$

is an orthogonal basis of $H^{2}(z) \ominus q_{1}(z) H^{2}(z)$.

Proof . It is easy to verify from the following:

$$
\begin{aligned}
& H^{2}(z) \ominus q_{1}(z) H^{2}(z) \\
= & \left\{H^{2}(z) \ominus b_{\lambda_{0}}^{k_{0}}(z) H^{2}(z)\right\} \oplus\left\{b_{\lambda_{0}}^{k_{0}}(z)\left(H^{2}(z) \ominus b_{\lambda_{1}}^{k_{1}}(z) H^{2}\right)\right\} \\
& \oplus \cdots \oplus\left\{\left(b_{\lambda_{0}}^{k_{0}}(z) \cdots b_{\lambda_{l-1}}^{k_{l-1}}(z)\right)\left(H^{2}(z) \ominus b_{\lambda_{l}}^{k_{l}}(z) H^{2}(z)\right)\right\} .
\end{aligned}
$$

Theorem 3.3. Let $q_{1}(z)$ be a finite Blaschke product having the following form:

$$
q_{1}(z)=\prod_{i=0}^{l}\left(\frac{z-\lambda_{i}}{1-\bar{\lambda}_{i} z}\right)^{k_{i}}=\prod_{i=0}^{l} b_{\lambda_{i}}^{k_{i}}(z),
$$


and $\left\{e_{i}^{(j)}\right\}$ be the basis obtained in Lemma 3.4. Suppose that $\mathcal{N}=H^{2} \ominus q_{1}(z) H^{2}$ or $\mathcal{N}=\left(H^{2}(z) \ominus q_{1}(z) H^{2}(z)\right) \otimes\left(H^{2}(w) \ominus q_{2}(w) H^{2}(w)\right)$ as in Theorem 2.1. Then, $A$ is in $\mathcal{A}^{\prime}$ if and only if there exists a finite set of bounded analytic functions $\left\{\varphi_{i}^{(j)}(w)\right\}$ such that $A=S_{\varphi}$ where $\varphi(z, w)=\sum_{i, j} e_{i}^{(j)}(z) \varphi_{i}^{(j)}(w)$.

Proof . It suffices to consider the case

$$
\mathcal{N}=\left(H^{2}(z) \ominus q_{1}(z) H^{2}(z)\right) \otimes\left(H^{2}(w) \ominus q_{2}(w) H^{2}(w)\right) .
$$

By Lemma 3.1, there is a function $\Phi$ in $\mathcal{N}$ such that $A=S_{\Phi}$. Since $\Phi$ is in $\mathcal{N}$, we have the following finite expansion of $\Phi$ :

$$
\Phi=\sum_{i, j} e_{i}^{(j)}(z) \phi_{i}^{(j)}(w)
$$

where $\left\{e_{i}^{(j)}\right\}$ is the basis of $H^{2}(z) \ominus q_{1}(z) H^{2}(z)$ obtained in Lemma 3.4, and every $\phi_{i}^{(j)}(w)$ is in $H^{2}(w) \ominus q_{2}(w) H^{2}(w)$. To prove our assertion, we use an induction.

First, for any bounded functions $f(w)$ and $g(w)$ in $H^{2}(w) \ominus q_{2}(w) H^{2}(w)$, $k_{\lambda_{0}}(z) f(w)$ and $k_{\lambda_{0}}(z) g(w)$ are in $\mathcal{N}$, and we have

$$
\begin{aligned}
& \left\langle A k_{\lambda_{0}}(z) f(w), k_{\lambda_{0}}(z) g(w)\right\rangle \\
= & \left\langle S_{\Phi} k_{\lambda_{0}}(z) f(w), k_{\lambda_{0}}(z) g(w)\right\rangle \\
= & \sum_{i, j}\left\langle e_{i}^{(j)}(z) \phi_{i}^{(j)}(w) k_{\lambda_{0}}(z) f(w), k_{\lambda_{0}}(z) g(w)\right\rangle \\
= & \sum_{i, j}\left\langle e_{i}^{(j)}(z) k_{\lambda_{0}}(z), k_{\lambda_{0}}(z)\right\rangle\left\langle\phi_{i}^{(j)}(w) f(w), g(w)\right\rangle \\
= & \left\langle e_{0}^{(0)}(z) k_{\lambda_{0}}(z), k_{\lambda_{0}}(z)\right\rangle\left\langle\phi_{0}^{(0)}(w) f(w), g(w)\right\rangle \\
= & \frac{1}{\left(1-\left|\lambda_{0}\right|^{2}\right)^{2}}\left\langle\phi_{0}^{(0)}(w) f(w), g(w)\right\rangle .
\end{aligned}
$$

Hence we have

$$
\left|\frac{1}{\left(1-\left|\lambda_{0}\right|^{2}\right)^{2}}\left\langle\phi_{0}^{(0)}(w) f(w), g(w)\right\rangle\right| \leq\|A\|\left\|k_{\lambda_{0}}(z)\right\|^{2}\|f(w)\|\|g(w)\|,
$$

furthermore

$$
\left|\left\langle\phi_{0}^{(0)}(w) f(w), g(w)\right\rangle\right| \leq\left(1-\left|\lambda_{0}\right|^{2}\right)\|A\|\|f(w)\|\|g(w)\| .
$$

By Theorem 1 in [8], there exists a bounded analytic function $\varphi_{0}^{(0)}$, we can substitute $\varphi_{0}^{(0)}(w)$ for $\phi_{0}^{(0)}(w)$, that is, $S_{\phi_{0}^{(0)}(w)}=S_{\varphi_{0}^{(0)}(w)}$.

Next, for $0 \leq i \leq m, 0 \leq j \leq k_{i}-1(i<m)$ and $0 \leq j \leq n \leq k_{m}-1(i=m)$, we assume that each $\varphi_{i}^{(j)}(w)$ is a bounded analytic function. Then

$$
A_{m, n}:=S_{\Phi}-\sum^{\prime} S_{e_{i}^{(j)} \varphi_{i(j)}}
$$

is a bounded operator on $\mathcal{N}$, where the sum $\sum^{\prime}$ is taken only over $0 \leq i \leq m$, $0 \leq j \leq k_{i}-1(i<m)$ and $0 \leq j \leq n(i=m)$. 
If $n<k_{m}-1$, then

$$
\begin{aligned}
& \left\langle A_{m, n} k_{\lambda_{m}}(z) f(w), k_{\lambda_{m}}(z) b_{\lambda_{m}}^{n+1}(z) g(w)\right\rangle \\
= & \sum^{\prime \prime}\left\langle e_{i}^{(j)}(z) \phi_{i}^{(j)}(w) k_{\lambda_{m}}(z) f(w), k_{\lambda_{m}}(z) b_{\lambda_{m}}^{n+1}(z) g(w)\right\rangle \\
= & \sum^{\prime \prime}\left\langle e_{i}^{(j)}(z) k_{\lambda_{m}}(z), k_{\lambda_{m}}(z) b_{\lambda_{m}}^{n+1}(z)\right\rangle\left\langle\phi_{i}^{(j)}(w) f(w), g(w)\right\rangle \\
= & \left\langle e_{m}^{(n+1)}(z) k_{\lambda_{m}}(z), k_{\lambda_{m}}(z) b_{\lambda_{m}}^{n+1}(z)\right\rangle\left\langle\phi_{m}^{(n+1)}(w) f(w), g(w)\right\rangle \\
= & \frac{1}{\left(1-\left|\lambda_{m}\right|^{2}\right)^{2}} b_{\lambda_{0}}^{k_{0}}\left(\lambda_{m}\right) \cdots b_{\lambda_{m}-1}^{k_{m-1}}\left(\lambda_{m}\right)\left\langle\phi_{m}^{(n+1)}(w) f(w), g(w)\right\rangle,
\end{aligned}
$$

where the sum $\sum^{\prime \prime}$ is taken over $m \leq i \leq l, n+1 \leq j \leq k_{m}-1(i=m)$ and $0 \leq j \leq k_{i}-1(i>m)$. Hence we have

$$
\left|\left\langle\phi_{m}^{(n+1)}(w) f(w), g(w)\right\rangle\right| \leq K_{m, n}\left\|A_{m, n}\right\|\|f(w)\|\|g(w)\|,
$$

for some constant $K_{m, n}$. Therefore, by Theorem 1 in [8], there exists a bounded analytic function $\varphi_{m}^{(n+1)}$, we can substitute $S_{\varphi_{m}^{(n+1)}(w)}$ for $S_{\phi_{m}^{(n+1)}(w)}$.

If $n=k_{m}-1$, then

$$
\begin{aligned}
& \left\langle A_{m, n} k_{\lambda_{m+1}}(z) f(w), k_{\lambda_{m+1}}(z) g(w)\right\rangle \\
= & \sum^{\prime \prime \prime}\left\langle e_{i}^{(j)}(z) \phi_{i}^{(j)}(w) k_{\lambda_{m+1}}(z) f(w), k_{\lambda_{m+1}}(z) g(w)\right\rangle \\
= & \sum^{\prime \prime \prime}\left\langle e_{i}^{(j)}(z) k_{\lambda_{m+1}}(z), k_{\lambda_{m+1}}(z)\right\rangle\left\langle\phi_{i}^{(j)}(w) f(w), g(w)\right\rangle \\
= & \left\langle e_{m+1}^{(0)}(z) k_{\lambda_{m+1}}(z), k_{\lambda_{m+1}}(z)\right\rangle\left\langle\phi_{m+1}^{(0)}(w) f(w), g(w)\right\rangle \\
= & \frac{1}{\left(1-\left|\lambda_{m+1}\right|^{2}\right)^{2}} b_{\lambda_{0}}^{k_{0}}\left(\lambda_{m+1}\right) \cdots b_{\lambda_{m-1}}^{k_{m}}\left(\lambda_{m+1}\right)\left\langle\phi_{m+1}^{(0)}(w) f(w), g(w)\right\rangle,
\end{aligned}
$$

where the sum $\sum^{\prime \prime \prime}$ is taken over $m+1 \leq i \leq l$ and $0 \leq j \leq k_{i}-1$. Hence we have

$$
\left|\left\langle\phi_{m+1}^{(0)}(w) f(w), g(w)\right\rangle\right| \leq K_{m, n}^{\prime}\left\|A_{m, n}\right\|\|f(w)\|\|g(w)\|,
$$

for some constant $K_{m, n}^{\prime}$. Therefore, by Theorem 1 in [8], there exists a bounded analytic function $\varphi_{m+1}^{(0)}(w)$, we can substitute $S_{\varphi_{m+1}^{(0)}(w)}$ for $S_{\phi_{m+1}^{(0)}(w)}$. Therefore $\varphi(z, w)=\sum_{i, j} e_{i}^{(j)}(z) \varphi_{i}^{(j)}(w)$ is a bounded analytic function and $S_{\Phi}=S_{\varphi}$. and $S_{w}$.

Conversely, it is easy to check that if $\varphi$ is in $H^{\infty}$ then $S_{\varphi}$ commutes with $S_{z}$

Corollary 3.2. If $\mathcal{N}_{z}$ is of finite dimension, then $\mathcal{A}=\mathcal{A}^{\prime}$ on $\mathcal{N}=\mathcal{N}_{z} \otimes \mathcal{N}_{w}$. 


\section{Norm estimates}

In this section, we deal with norm estimates of $S_{\varphi}$. To begin with, we should recall the one-variable case, in which the following norm equality was obtained by Sarason in Proposition 2.1 in [8]:

$$
\left\|S_{\varphi(z)}\right\|=\left\|\varphi(z)+q(z) H^{\infty}(z)\right\|_{\infty} \text { on } \mathcal{N}_{z}=H^{2}(z) \ominus q(z) H^{2}(z),
$$

where $H^{\infty}(z)$ is the Banach algebra of all bounded analytic functions with the variable $z$ on $\mathbb{D}, \varphi(z)$ is in $H^{\infty}(z)$ and $q(z)$ is an inner function in $H^{\infty}(z)$.

Let $H_{f}$ denote the Hankel operator of a bounded function $f$, that is, $H_{f}=$ $\left.\left(I_{L^{2}}-P_{H^{2}}\right) M_{f}\right|_{H^{2}}$, where $M_{f}$ is the multiplication operator by $f$ on $L^{2}$.

The following lemma is known for the one-variable case.

Lemma 4.1. Suppose that $\mathcal{N}=H^{2} \ominus q H^{2}$ for an inner function $q$. Then $M_{q} H_{\bar{q} \varphi} P_{\mathcal{N}}=S_{\varphi} P_{\mathcal{N}}$ for any $\varphi$ in $H^{\infty}$ and $\left\|H_{\bar{q} \varphi}\right\|=\left\|S_{\varphi}\right\|$.

Proof. Since

$$
\begin{aligned}
M_{q} H_{\bar{q} \varphi} P_{\mathcal{N}} f=q\left(I_{L^{2}}-P_{H^{2}}\right) \bar{q} \varphi P_{\mathcal{N}} f & =\left(I_{L^{2}}-q P_{H^{2}} \bar{q}\right) \varphi P_{\mathcal{N}} f \\
& =\left(P_{H^{2}}-q P_{H^{2}} \bar{q}\right) \varphi P_{\mathcal{N}} f \\
& =P_{\mathcal{N}} \varphi P_{\mathcal{N}} f \\
& =S_{\varphi} P_{\mathcal{N}} f
\end{aligned}
$$

we have the first assertion. Next, we show the second one. It is easy to check that $q H^{2} \subseteq \operatorname{ker} H_{\bar{q} \varphi}$, we have $\left\|S_{\varphi}\right\|=\left\|S_{\varphi} P_{\mathcal{N}}\right\|=\left\|M_{q} H_{\bar{q} \varphi} P_{\mathcal{N}}\right\|=\left\|H_{\bar{q} \varphi} P_{\mathcal{N}}\right\|=\left\|H_{\bar{q} \varphi}\right\|$.

The next lemma is elementary.

Lemma 4.2. Let $X$ be $a \mathrm{w}^{*}$-closed subspace of $L^{\infty}$. Then, for any $f$ in $L^{\infty}$, there exists a function $F$ in $f+X$ such that $\|F\|_{\infty} \leq\|f+X\|_{\infty}=\inf _{g \in X}\|f+g\|_{\infty}$.

Proof . We set $\alpha=\|f+X\|_{\infty}$. For any $n$ in $\mathbb{N}$, there exists a function $f_{n}$ in $f+X$ such that $\left\|f_{n}\right\|_{\infty} \leq \alpha+1 / n$. Since $\left\|f_{n}\right\|_{\infty} \leq \alpha+1,\left\{f_{n}\right\}_{n \in \mathbb{N}}$ is $\mathrm{w}^{*}$-compact in $L^{\infty}$. Hence there exists a subsequence $\left\{f_{n_{k}}\right\}_{k}$ such that $f_{n_{k}}$ converges to some function $F$ in the $\mathrm{w}^{*}$-topology of $L^{\infty}$ as $k$ tends to $+\infty$. Then $\|F\|_{\infty} \leq \liminf _{k \in \mathbb{N}}\left\|f_{n_{k}}\right\|_{\infty} \leq$ $\liminf \operatorname{in}_{k \in \mathbb{N}}\left(\alpha+1 / n_{k}\right)=\alpha$ and $F$ is in $f+X$.

Let $\mathbb{H}_{z}^{\infty}$ (resp. $\left.\mathbb{H}_{w}^{\infty}\right)$ denote the $\mathrm{w}^{*}$-closed subalgebra of $L^{\infty}$ generated by $z$, $w$ and $\bar{w}$ (resp. $z, \bar{z}$ and $w$ ).

Theorem 4.1. Let $q_{1}(z)$ be an inner function depending only on the variable $z$ and let $\mathfrak{Z}(f(z))$ be the set of all zero points of a one-variable function $f(z)$ in $\mathbb{D}$, that is, $\mathfrak{Z}(f(z))=\{\lambda \in \mathbb{D}: f(\lambda)=0\}$. Suppose that $\varphi$ is in $H^{\infty}$. Then the following norm estimates hold:

(i) Suppose that $\mathcal{N}=H^{2} \ominus q_{1}(z) H^{2}$ and let $I_{1}$ denote

$$
\max \left\{\sup _{\lambda \in \mathbb{D}}\left\|\varphi(z, \lambda)+q_{1}(z) H^{\infty}(z)\right\|_{\infty}, \sup _{\lambda \in \mathcal{Z}\left(q_{1}\right)}\|\varphi(\lambda, w)\|_{\infty}\right\} .
$$


Then

$$
I_{1} \leq\left\|S_{\varphi}\right\|=\left\|\varphi+q_{1}(z) \mathbb{H}_{z}^{\infty}\right\|_{\infty} .
$$

(ii) Suppose that $\mathcal{N}=\left(H^{2}(z) \ominus q_{1}(z) H^{2}(z)\right) \otimes\left(H^{2}(w) \ominus q_{2}(w) H^{2}(w)\right)$ for an inner function $q_{2}(w)$ depending only on the variable $w$ and let $I_{2}$ denote

$$
\max \left\{\sup _{\lambda \in \mathfrak{Z}\left(q_{1}\right)}\left\|\varphi(\lambda, w)+q_{2}(w) H^{\infty}(w)\right\|_{\infty}, \sup _{\lambda \in \mathfrak{Z}\left(q_{2}\right)}\left\|\varphi(z, \lambda)+q_{1}(z) H^{\infty}(z)\right\|_{\infty}\right\} .
$$

Then

$$
I_{2} \leq\left\|S_{\varphi}\right\| \leq\left\|\varphi+\left(q_{1}(z) H^{2}+q_{2}(w) H^{2}\right) \cap H^{\infty}\right\|_{\infty} .
$$

Proof. We show the assertion of (i). By Lemma 4.1, $\left\|S_{\varphi}\right\|=\left\|H_{\overline{q_{1}} \varphi}\right\|$. Since $\left\|H_{\overline{q_{1}} \varphi}\right\|=\left\|\overline{q_{1}(z)} \varphi+\mathbb{H}_{z}^{\infty}\right\|$ by Corollary 1 in $[6]$, we have $\left\|S_{\varphi}\right\|=\left\|\overline{q_{1}(z)} \varphi+\mathbb{H}_{z}^{\infty}\right\|$. Hence we have the equality in (i).

Next, for any $\lambda$ in $\mathbb{D}$, any $f_{1}$ and $g_{1}$ in $H^{2}(z) \ominus q_{1}(z) H^{2}(z)$,

$$
\begin{aligned}
\left|\int \varphi(z, \lambda) f_{1}(z) \overline{g_{1}(z)} d \sigma_{1}\right| & =\left|\int\left(\int \varphi(z, w) e_{\lambda}(w) \overline{e_{\lambda}(w)} d|w|\right) f_{1}(z) \overline{g_{1}(z)} d \sigma_{1}\right| \\
& =\left|\iint \varphi(z, w) f_{1}(z) e_{\lambda}(w) \overline{g_{1}(z) e_{\lambda}(w)} d \sigma\right| \\
& =\left|\left\langle S_{\varphi} f_{1}(z) e_{\lambda}(w), g_{1}(z) e_{\lambda}(w)\right\rangle\right| \\
& \leq\left\|S_{\varphi}\right\|\left\|f_{1}\right\|\left\|g_{1}\right\|,
\end{aligned}
$$

where $e_{\lambda}(w)=\left(1-|\lambda|^{2}\right)^{1 / 2}(1-\bar{\lambda} w)$. Hence

$$
\left\|S_{\varphi}\right\| \geq\left\|S_{\varphi(\cdot, \lambda)}\right\|=\left\|\varphi(z, \lambda)+q_{1}(z) H^{\infty}(z)\right\|,
$$

for any $\lambda$ in $\mathbb{D}$ by Proposition 2.1 in [8].

Let $\lambda$ be in $\mathfrak{Z}\left(q_{1}\right)$. Then $e_{\lambda}(z)$ is in $H^{2}(z) \ominus q_{1}(z) H^{2}(z)$. For any $f_{2}$ and $g_{2}$ in $H^{2}(w)$, by the same calculation as the above,

$$
\left|\int \varphi(\lambda, w) f_{2}(w) \overline{g_{2}(w)} d\right| w|| \leq\left\|S_{\varphi}\right\|\left\|f_{2}\right\|\left\|g_{2}\right\| \text {. }
$$

Hence

$$
\left\|S_{\varphi}\right\| \geq\left\|T_{\varphi(\lambda, \cdot)}\right\|=\|\varphi(\lambda, w)\| .
$$

(4.1) and (4.2) conclude (i)

By calculations similar to those in the proof of (i), we have inequalities in (ii).

\section{5. $\left(q_{1} H^{2}+q_{2} H^{2}\right) \cap H^{\infty}=q_{1} H^{\infty}+q_{2} H^{\infty}$ ?}

In this section, we study the following problem (cf. [5]):

$$
\left(q_{1}(z) H^{2}+q_{2}(w) H^{2}\right) \cap H^{\infty}=q_{1}(z) H^{\infty}+q_{2}(w) H^{\infty} ?
$$

Theorem 5.1. If $q_{1}(z)$ is a finite Blaschke product, then the equality (5.1) holds. 
Proof . Let $\operatorname{deg}\left(q_{1}(z)\right)=k<+\infty$. Since

$$
q_{1}(z) H^{2}+q_{2}(w) H^{2}=q_{1}(z) H^{2} \oplus\left(H^{2}(z) \ominus q_{1}(z) H^{2}(z)\right) \otimes q_{2}(w) H^{2}(w),
$$

we have $f=q_{1}(z) g \oplus q_{2}(w) \sum_{i=1}^{k} \oplus e_{i}(z) h_{i}(w)$ for any $f$ in $\left(q_{1}(z) H^{2}+q_{2}(w) H^{2}\right) \cap$ $H^{\infty}$, where $\left\{e_{i}\right\}_{i=1}^{k}$ is an orthonormal basis of $H^{2}(z) \ominus q_{1}(z) H^{2}(z), g$ is in $H^{2}$ and every $h_{i}(w)$ is in $H^{2}(w)$. We note that every $e_{i}(z)$ is a bounded rational function. Since

$$
\begin{aligned}
\int_{\mathbb{T}} f \overline{e_{i}(z)} d \sigma_{1} & =\int\left(q_{1}(z) g \oplus q_{2}(w) \sum_{j=1}^{k} e_{j}(z) h_{j}(w)\right) \overline{e_{i}(z)} d \sigma_{1} \\
& =\int q_{1}(z) g \overline{e_{i}(z)} d \sigma_{1}+\sum_{j=1}^{k} q_{2}(w) h_{j}(w)\left\langle e_{j}(z), e_{i}(z)\right\rangle_{H^{2}(z)} \\
& =q_{2}(w) h_{i}(w),
\end{aligned}
$$

we have

$$
\left|h_{i}(w)\right|=\left|q_{2}(w) h_{i}(w)\right|=\left|\int f \overline{e_{i}(z)} d \sigma_{1}\right| \leq\|f\|_{\infty}\left\|e_{i}(z)\right\|_{\infty} .
$$

Therefore every $h_{i}(w)$ and $g$ are bounded. Hence $\left(q_{1}(z) H^{2}+q_{2}(w) H^{2}\right) \cap H^{\infty} \subseteq$ $q_{1}(z) H^{\infty}+q_{2}(w) H^{\infty}$. Trivially, $\left(q_{1}(z) H^{2}+q_{2}(w) H^{2}\right) \cap H^{\infty} \supseteq q_{1}(z) H^{\infty}+q_{2}(w) H^{\infty}$. Hence (5.1) holds.

Next we give another approach to this problem. Consider two bounded linear maps $\delta_{1}$ and $\delta_{2}$ defined as follows:

$$
\begin{aligned}
& \delta_{1} \quad: \quad H^{2} \rightarrow H^{2} \oplus H^{2}, \delta_{1}(f)=\left(-q_{2}(w) f, q_{1}(z) f\right) \\
& \delta_{2} \quad: \quad H^{2} \oplus H^{2} \rightarrow q_{1}(z) H^{2}+q_{2}(w) H^{2}, \delta_{2}(f, g)=q_{1}(z) f+q_{2}(w) g .
\end{aligned}
$$

Then the following sequence is exact:

$$
0 \longrightarrow H^{2} \stackrel{\delta_{1}}{\longrightarrow} H^{2} \oplus H^{2} \stackrel{\delta_{2}}{\longrightarrow} q_{1}(z) H^{2}+q_{2}(w) H^{2} \longrightarrow 0 .
$$

Indeed, it is easy to check that $\operatorname{Im} \delta_{1} \subseteq \operatorname{ker} \delta_{2}$. We show that $\operatorname{ker} \delta_{2} \subseteq \operatorname{Im} \delta_{1}$. Let $(f, g)$ be in $\operatorname{ker} \delta_{2}$. Then

$$
\left\langle\left(q_{1}(z) f+q_{2}(w) g\right) F, G\right\rangle=\langle 0, G\rangle=0
$$

for any $F$ and $G$ in $H^{2}$. Set $F=1$ and let $G$ be an element in $H^{2} \ominus q_{1}(z) H^{2}$. Then

$$
\left\langle\left(q_{1}(z) f+q_{2}(w) g\right) 1, G\right\rangle=\left\langle q_{2}(w) g, G\right\rangle=0 .
$$

Hence $q_{2}(w) g$ is in $q_{1}(z) H^{2}$, that is, $q_{2}(w) g=q_{1}(z) g^{\prime}$ for some function $g^{\prime}$ in $H^{2}$. Furthermore, $g=T_{q_{2}(w)}^{*} q_{2}(w) g=T_{q_{2}(w)}^{*} q_{1}(z) g^{\prime}=q_{1}(z) T_{q_{2}(w)}^{*} g^{\prime}$. Setting $h=$ $T_{q_{2}(w)}^{*} g^{\prime}$, we have $0=q_{1}(z) f+q_{2}(w) g=q_{1}(z) f+q_{2}(w)\left(q_{1}(z) h\right)$ and $f=-q_{2}(w) h$. Thus $(f, g)=\left(-q_{2}(w) h, q_{1}(z) h\right)=\delta_{1}(h)$.

Thus, $\varphi=q_{1}(z) f_{1}+q_{2}(w) g_{1}=q_{1}(z) f_{2}+q_{2}(w) g_{2}$ if and only if there exists a function $h$ in $H^{2}$ such that $f_{1}-f_{2}=-q_{2}(w) h$ and $g_{1}-g_{2}=q_{1}(z) h$. By this observation we have the following: 
Theorem 5.2. The equality (5.1) holds if and only if, for any function $\varphi$ in $H^{\infty}$ having a form $\varphi=q_{1}(z) f+q_{2}(w) g$ for some functions $f$ and $g$ in $H^{2}$, there exists a function $h$ in $H^{2}$ such that both $f-q_{2}(w) h$ and $g+q_{1}(z) h$ are bounded.

\section{A Carathéodory type interpolation problem in two variables}

In this section, we consider the following interpolation problem:

- Given $n+1$ analytic functions $u_{0}(z), \ldots, u_{n}(z)$ in $H^{\infty}(z)$, can one find a function $\varphi$ in $H^{\infty}$ whose power series begins with $u_{0}(z)+\cdots+u_{j}(z) w^{j}+$ $\cdots+u_{n}(z) w^{n}$ and $\|\varphi\|_{\infty} \leq 1$ ?

We set $\mathcal{N}=H^{2} / w^{n+1} H^{2}=H^{2}(z) \otimes \mathcal{N}_{w}$. Then $\mathcal{N}$ can be identified with $H^{2}(z) \oplus \cdots \oplus H^{2}(z)$ (the direct sum of $n+1$ copies of $\left.H^{2}(z)\right)$. Assume that an operator $T$ on $\mathcal{N}$ commutes with both $T_{z}$ and $S_{w}$. Then

$$
T\left(\begin{array}{ccc}
T_{z} & & 0 \\
& \ddots & \\
0 & & T_{z}
\end{array}\right)=\left(\begin{array}{ccc}
T_{z} & & 0 \\
& \ddots & \\
0 & & T_{z}
\end{array}\right) T \text { on } H^{2}(z) \oplus \cdots \oplus H^{2}(z) .
$$

Hence $T_{i j} T_{z}=T_{z} T_{i j}$ for $T=\left(T_{i j}\right)$. Then, for every $T_{i j}$, there exists a function $\varphi_{i j}(z)$ in $H^{\infty}(z)$ such that $T_{\varphi_{i j}(z)}=T_{i j}$. Since $T$ commutes with $S_{w}$, that is, $T$ commutes with the truncated shift operator on $\mathcal{N}_{w}$ with respect to the orthonormal basis $\left\{1, \ldots, w^{n}\right\}, T$ has the following form on $H^{2}(z) \oplus \cdots \oplus H^{2}(z)$ :

$$
T=C\left(T_{\varphi_{0}(z)}, \ldots, T_{\varphi_{n}(z)}\right):=\left(\begin{array}{ccccc}
T_{\varphi_{0}(z)} & 0 & 0 & \cdots & 0 \\
T_{\varphi_{1}(z)} & T_{\varphi_{0}(z)} & 0 & \cdots & 0 \\
\cdot & \cdot & \cdot & \cdots & \cdot \\
T_{\varphi_{n}(z)} & T_{\varphi_{n-1}(z)} & T_{\varphi_{n-2}(z)} & \cdots & T_{\varphi_{0}(z)}
\end{array}\right),
$$

where $\varphi_{j}(z)$ denotes $\varphi_{j 0}(z)$, for short.

Theorem 6.1. Let $u_{0}(z), \ldots, u_{n}(z)$ be given $n+1$ analytic functions in $H^{\infty}(z)$. Then $\left\|C\left(T_{u_{0}(z)}, \ldots, T_{u_{n}(z)}\right)\right\| \leq 1$ if and only if there exists a function $\psi$ in $\mathbb{H}_{w}^{\infty}$ whose power series begins with $u_{0}(z)+\cdots+u_{j}(z) w^{j}+\cdots+u_{n}(z) w^{n}$ and $\|\psi\|_{\infty} \leq 1$.

Proof. Suppose that $u_{0}(z), \ldots, u_{n}(z)$ are $n+1$ analytic functions in $H^{\infty}(z)$. We set $\mathcal{N}=H^{2} /\left(w^{n+1} H^{2}\right)=H^{2}(z) \otimes\left(H^{2}(w) \ominus w^{n+1} H^{2}(w)\right)$. Then $T=$ $C\left(T_{u_{0}(z)}, \ldots, T_{u_{n}(z)}\right)$ is a bounded linear operator on $H^{2}(z) \oplus \cdots \oplus H^{2}(z)$ (the direct sum of $n$ copies of $\left.H^{2}(z)\right)$. It is easy to check $T$ commutes with $T_{z}$ and $S_{w}$ Setting $\varphi=T \cdot 1$, we have $\varphi=u_{0}(z)+\cdots+u_{n}(z) w^{n}$ and we showed $T=S_{\varphi}$ in the proof of Lemma 3.1.

If $\|T\| \leq 1$ then $\left\|S_{\varphi}\right\| \leq 1$. Since $\left\|S_{\varphi}\right\|=\left\|\varphi+w^{n+1} \mathbb{H}_{w}^{\infty}\right\|$ by (i) in Theorem 4.1 , there exists a function in $\psi$ in $\mathbb{H}_{w}^{\infty}$ such that $\|\psi\|_{\infty} \leq 1$. Then the power series of $\psi$ begins with $u_{0}(z)+\cdots+u_{n}(z) w^{n}$.

Conversely, if there exists a function $\psi$ in $\mathbb{H}_{w}^{\infty}$ such that whose power series begins with $u_{0}(z)+\cdots+u_{n}(z) w^{n}$ and $\|\psi\|_{\infty} \leq 1$. Then $\psi-\varphi$ is in $w^{n+1} \mathbb{H}_{w}^{\infty}$ where we set $\varphi=u_{0}(z)+\cdots+u_{n}(z) w^{n},\left\|S_{\varphi}\right\|=\left\|\varphi+w^{n+1} \mathbb{H}_{w}^{\infty}\right\|_{\infty} \leq\|\psi\|_{\infty} \leq$ 
1 by (i) in Theorem 4.1 and $S_{\varphi}$ can be identified with $C\left(T_{u_{0}(z)}, \ldots, T_{u_{n}(z)}\right)$ on $H^{2}(z) \oplus \cdots \oplus H^{2}(z)$. This completes the proof.

\section{A Nevanlinna-Pick type interpolation problem in two variables}

In this section we study the following interpolation problem:

- Let $\lambda_{1}, \ldots, \lambda_{n}$ be $n$ distinct points in $\mathbb{D}$ and $u_{1}(w), \ldots, u_{n}(w)$ be $n$ analytic functions in $H^{\infty}(w)$. Then, can one find a function $\varphi$ in $H^{\infty}$ such that $\|\varphi\|_{\infty} \leq 1$ and $\varphi\left(\lambda_{j}, w\right)=u_{j}(w)$ for $j=1, \ldots, n$ ?

To begin with, we prove the following lemma:

Lemma 7.1. Let $\lambda_{1}, \ldots, \lambda_{n}$ be $n$ points in $\mathbb{D}$ and let $\varphi$ be a function in $\mathbb{H}_{z}^{\infty}$. If $\varphi\left(\lambda_{j}, w\right)=0$ w-a.e. for every $\lambda_{j}$ then $\varphi$ is a function in $q(z) \mathbb{H}_{z}^{\infty}$, where $q(z)=$ $\prod_{j=1}^{n} b_{\lambda_{j}}(z)$.

Proof . For any integer $l$,

$$
\varphi_{l}(z):=\int_{\mathbb{T}} \varphi(z, w) \bar{w}^{l} d \sigma_{2}
$$

can be extended analytically to $\mathbb{D}$ with respect to the variable $z$. Since $\varphi\left(\lambda_{j}, w\right)=0$ $w$-a.e.,

$\varphi_{l}\left(\lambda_{j}\right)=\int_{\mathbb{T}} \varphi_{l}(z) \overline{k_{\lambda_{j}}(z)} d \sigma_{1}=\int_{\mathbb{T}} \int_{\mathbb{T}} \varphi(z, w) \overline{k_{\lambda_{j}}(z) w^{l}} d \sigma=\int_{\mathbb{T}} \varphi\left(\lambda_{j}, w\right) \bar{w}^{l} d \sigma_{2}=0$.

Hence there exists a function $\psi(z)$ in $H^{2}(z)$ such that $\varphi_{l}(z)=q(z) \psi_{l}(z)$. Therefore

$$
\varphi=\sum_{l \in \mathbb{Z}} \varphi_{l}(z) w^{l}=\sum_{l \in \mathbb{Z}} q(z) \psi_{l}(z) w^{l}=q(z) \sum_{l \in \mathbb{Z}} \psi_{l}(z) w^{l} .
$$

Therefore $\varphi$ is in $q(z) \mathbb{H}_{z}^{2}$. Since $\varphi / q(z)$ is a bounded function on $\mathbb{T}^{2}$, we have the conclusion.

Theorem 7.1. Let $\lambda_{1}, \ldots, \lambda_{n}$ be $n$ distinct points in $\mathbb{D}$ and $u_{1}(w), \ldots, u_{n}(w)$ be $n$ functions in $H^{\infty}(w)$ such that $\left\|u_{j}(w)\right\|_{\infty} \leq 1$ for $j=1, \ldots, n$. Then the quadratic form

$$
\sum_{1 \leq i, j \leq n} \frac{\left\langle\left(1-T_{u_{j}(w)} T_{u_{i}(w)}^{*}\right) f_{i}(w), f_{j}(w)\right\rangle}{1-\overline{\lambda_{i}} \lambda_{j}}
$$

is non-negative for any $f_{1}(w), \ldots, f_{n}(w)$ in $H^{2}(w)$ if and only if there exists a function $\varphi$ in $\mathbb{H}_{z}^{\infty}$ satisfying $\|\varphi\|_{\infty} \leq 1$ and $\varphi\left(\lambda_{j}, w\right)=u_{j}(w)$ for $j=1, \ldots, n$.

Proof . We define an operator as follows:

$$
A^{*} k_{\lambda_{i}}(z) f(w)=k_{\lambda_{i}}(z) T_{u_{i}(w)}^{*} f(w),
$$


for every $0 \leq i \leq n$ and $f(w)$ in $H^{2}(w)$. Then $A$ is a bounded linear operator on $\left(H^{2}(z) \ominus q(z) H^{2}(z)\right) \otimes H^{2}(w)$, where $q(z)=\prod_{i=1}^{n} b_{\lambda_{i}}(z)$. Since every $T_{u_{i}(w)}^{*}$ commutes with $T_{w}^{*}$ and

$$
\begin{aligned}
S_{z}^{*} A^{*} k_{\lambda_{i}}(z) f(w) & =S_{z}^{*} k_{\lambda_{i}}(z) T_{u_{i}(w)}^{*} f(w) \\
& =\overline{\lambda_{i}} k_{\lambda_{i}}(z) T_{u_{i}(w)}^{*} f(w) \\
& =A^{*} \overline{\lambda_{i}} k_{\lambda_{i}}(z) f(w) \\
& =A^{*} S_{z}^{*} k_{\lambda_{i}}(z) f(w)
\end{aligned}
$$

Hence $A$ commutes with $S_{z}$ and $T_{w}$. By Theorem 3.3, there exists $\psi$ in $H^{\infty}$ such that $A=S_{\psi}$, and we have $\psi\left(\lambda_{i}, w\right)=u_{i}(w)$. Indeed,

$$
\begin{aligned}
\left\langle S_{\psi} k_{\lambda_{i}}(z) f(w), k_{\lambda_{j}}(z) g(w)\right\rangle & =\left\langle\psi(z, w) k_{\lambda_{i}}(z) f(w), k_{\lambda_{j}}(z) g(w)\right\rangle \\
& =\left\langle k_{\lambda_{i}}\left(\lambda_{j}\right) \psi\left(\lambda_{j}, w\right) f(w), g(w)\right\rangle
\end{aligned}
$$

and

$$
\begin{aligned}
\left\langle S_{\psi} k_{\lambda_{i}}(z) f(w), k_{\lambda_{j}}(z) g(w)\right\rangle & =\left\langle A k_{\lambda_{i}}(z) f(w), k_{\lambda_{j}}(z) g(w)\right\rangle \\
& =\left\langle k_{\lambda_{i}}(z) f(w), A^{*} k_{\lambda_{j}}(z) g(w)\right\rangle \\
& =\left\langle k_{\lambda_{i}}(z) f(w), k_{\lambda_{j}}(z) T_{u_{j}(w)}^{*} g(w)\right\rangle \\
& =\left\langle k_{\lambda_{i}}(z) T_{u_{j}(w)} f(w), k_{\lambda_{j}}(z) g(w)\right\rangle \\
& =\left\langle k_{\lambda_{i}}\left(\lambda_{j}\right) u_{j}(w) f(w), g(w)\right\rangle
\end{aligned}
$$

for any $f(w)$ and $g(w)$ in $H^{2}(w)$. Hence $\psi\left(\lambda_{i}, w\right)=u_{i}(w)$. Moreover

$$
\begin{aligned}
& \left\langle\left(I-A A^{*}\right) \sum_{i} k_{\lambda_{i}}(z) f_{i}(w), \sum_{j} k_{\lambda_{j}}(z) f_{j}(w)\right\rangle \\
= & \sum_{i, j} \frac{\left\langle f_{i}(w), f_{j}(w)\right\rangle}{1-\overline{\lambda_{i}} \lambda_{j}}-\sum_{i, j} \frac{\left\langle T_{u_{i}(w)}^{*} f_{i}(w), T_{u_{j}(w)}^{*} f_{j}(w)\right\rangle}{1-\overline{\lambda_{i}} \lambda_{j}} \\
= & \sum_{i, j} \frac{\left\langle\left(1-T_{u_{j}(w)} T_{u_{i}(w)}^{*}\right) f_{i}(w), f_{j}(w)\right\rangle}{1-\overline{\lambda_{i}} \lambda_{j}}
\end{aligned}
$$

Hence we have $\|A\| \leq 1$ if and only if the quadratic form

$$
\sum_{1 \leq i, j \leq n} \frac{\left\langle\left(1-T_{u_{j}(w)} T_{u_{i}(w)}^{*}\right) f_{i}(w), f_{j}(w)\right\rangle}{1-\overline{\lambda_{i}} \lambda_{j}}
$$

is non-negative for any $f_{1}(w), \ldots, f_{n}(w)$ in $H^{2}(w)$.

We suppose that $\|A\| \leq 1$. Since $\|A\|=\left\|S_{\psi}\right\|=\left\|\psi+q(z) \mathbb{H}_{z}^{\infty}\right\|_{\infty}$ by (i) in Theorem 4.1, there exists a function $\varphi$ in $\mathbb{H}_{z}^{\infty}$ such that $\|\varphi\|_{\infty} \leq 1$ and $\varphi$ is in $\psi+q(z) \mathbb{H}_{z}^{\infty}$. Then $\varphi\left(\lambda_{i}, w\right)=\psi\left(\lambda_{i}, w\right)=u_{j}(w)$ for $1 \leq i \leq n$. 
Conversely, we suppose that there exists a function $\varphi$ in $\mathbb{H}_{z}^{\infty}$ satisfying $\|\varphi\|_{\infty} \leq 1$ and $\varphi\left(\lambda_{j}, w\right)=u_{j}(w)$ for $1 \leq j \leq n$. Setting

$$
\psi(z, w)=\sum_{l=1}^{n} u_{l}(w) \prod_{j \neq l} \frac{\left(z-\lambda_{j}\right)}{\lambda_{l}-\lambda_{j}} \text { and } q(z)=\prod_{i=1}^{n} b_{\lambda_{i}}(z),
$$

we have $\varphi(z, w)-\psi(z, w)$ is an element of $q(z) \mathbb{H}_{z}^{\infty}$ by Lemma 7.1 and $\psi$ is in $H^{\infty}$. Let $A$ be the operator $S_{\psi}$ acting on $\left(H^{2}(z) \ominus q(z) H^{2}(z)\right) \otimes H^{2}(w)$. Then, by (i) in Theorem 4.1, we have

$$
\|A\|=\left\|S_{\psi}\right\|=\left\|\psi+q(z) \mathbb{H}_{z}^{\infty}\right\|_{\infty} \leq\|\varphi\|_{\infty} \leq 1 .
$$

Further, it is easy to check that

$$
A^{*} k_{\lambda_{i}}(z) f(w)=k_{\lambda_{i}}(z) T_{u_{i}(w)}^{*} f(w) .
$$

Hence the quadratic form

$$
\sum_{1 \leq i, j \leq n} \frac{\left\langle\left(1-T_{u_{j}(w)} T_{u_{i}(w)}^{*}\right) f_{i}(w), f_{j}(w)\right\rangle}{1-\overline{\lambda_{i}} \lambda_{j}}
$$

is non-negative for any $f_{1}(w), \ldots, f_{n}(w)$ in $H^{2}(w)$. This completes the proof.

\section{Acknowledgment}

The authors would like to thank the referee for his suggestions.

\section{References}

[1] J. Agler and J. E. McCarthy, Pick interpolation and Hilbert function spaces, Graduate Studies in Mathematics, Vol. 44, American Mathematical Society, Providence, RI, 2002.

[2] E. Amar and C. Menini, On an operator theory approach to the corona problem, Bull. London Math. Soc. 34 (2002), 369-373.

[3] D. N. Clark, On commuting contractions, J. Math. Anal. Appl. 32 (1970), 590-596.

[4] D. N. Clark, Commutants that do not dilate, Proc. Amer. Math. Soc. 35 No. 2 (1972), 483-486.

[5] K. Izuchi, T. Nakazi and M. Seto, Backward shift invariant subspaces in the bidisc II, J. Oper. Theory 51 (2004), 361-376.

[6] T. Nakazi, Toeplitz operators and weighted norm inequalities on the bidisc, Math. Inequal. Appl. Vol. 4, No. 3 (2001), 429-441.

[7] N. K. Nikolski, Operators, functions, and systems: an easy reading, Vol. 2, Model operators and systems, Mathematical Surveys and Monographs, Vol. 93, American Mathematical Society, Providence, RI, 2002.

[8] D. Sarason, Generalized interpolation in $H^{\infty}$, Trans. Amer. Math. Soc. 127 (1967), 179-203. 
[9] B. Sz.-Nagy and C. Foiaş, Harmonic analysis of operators on Hilbert space, North-Holland, Amsterdam, 1970.

Takahiko Nakazi and Michio Seto

Department of Mathematics

Faculty of Science

Hokkaido University

Sapporo 060-0810

Japan

e-mail: nakazi@math.sci.hokudai.ac.jp, seto@math.sci.hokudai.ac.jp

Current address of the second author

Department of Mathematics

Kanagawa University

Yokohama 221-8686, Japan

e-mail: seto@kanagawa-u.ac.jp 